\title{
Estereotipos y sesgos en el tratamiento de candidatas y candidatos en programas televisivos en las elecciones legislativas de Argentina en 2017
}

\section{Stereotypes and biases in the treatment of female and male candidates on television shows in the 2017 legislative elections in Argentina}

Virginia García Beaudoux. Universidad de Buenos Aires. Argentina.

virgarcia@ sociales.uba.ar

$[\mathrm{CV}] \bigcirc \bar{C}$

Orlando D'Adamo. Universidad de Buenos Aires. Argentina.

orlando.dadamo@ub.edu.ar

$[\mathrm{CV}]$ (1) $\mathrm{G}$

Salomé Berrocal Gonzalo. Universidad de Valladolid. España.

salomeb@hmca.uva.es

$[\mathrm{CV}] \odot \mathrm{G}$

Marina Gavensky. Universidad de Belgrano. Argentina.

gavenskym@gmail.com

$[\mathrm{CV}](\mathrm{C})$

Este artículo forma parte del proyecto de investigación CSO2017-84472-R: "Politainment en el entorno de la posverdad: nuevas narrativas, clickbait y gamificación" (POLITGAMENT), financiado por el Ministerio de Economía y Competitividad.

Fechas:

-inicio de la investigación: 1 de enero de 2018

-término de la investigación: 31 de diciembre de 2020

Cómo citar este artículo / Referencia normalizada

García Beaudoux, V., D’Adamo, O., Berrocal Gonzalo, S. y Gavensky, M. (2020). Estereotipos y sesgos en el tratamiento de candidatas y candidatos en programas televisivos en las elecciones legislativas de Argentina en 2017. Revista Latina de Comunicación Social, (77), 275-293. https://www.doi.org/10.4185/RLCS-2020-1458

\section{RESUMEN}

Introducción: El artículo se incluye en el marco de las investigaciones empíricas sobre la cobertura noticiosa en campaña electoral, con la novedad de examinar los estereotipos de género y sesgos en programas informativos y de infoentretenimiento político en las elecciones legislativas de Argentina en 2017. Metodología: Se realiza un análisis de contenido de los programas informativos y de politainment de mayor audiencia. La muestra está formada por 132 emisiones. Resultados: Se 
observa como los candidatos triplican en presencia a las candidatas en el conjunto de los programas examinados, además de recibir mayor número de menciones. En el caso de las mujeres los comentarios y preguntas que se les formulan se corresponden mayoritariamente con soft news mientras que a los hombres se les relacionan con hard news. Conclusiones: Los candidatos reciben más menciones e invitaciones en informativos y programas de entrevistas y predominan en las hard news. En las candidatas siguen presentes estereotipos de género relacionados con su apariencia física o rol doméstico.

PALABRAS CLAVE: soft news; hard news; infoentretenimiento; campaña electoral; Argentina; estereotipos de género.

\begin{abstract}
Introduction: The article is included in the framework of empirical research on news coverage in the electoral campaign, with the novelty of examining gender stereotypes and biases in information and political infotainment shows in the legislative elections in Argentina in 2017. Methodology: Content analysis is carried out of the most popular information and politainment shows. The sample is made up of 132 broadcasts. Results: It is observed how the presence of male candidates tripled the presence of female candidates in all the examined shows, besides receiving a greater number of mentions. In the case of women, the comments and questions made to them correspond mostly to soft news, while men are related to hard news. Conclusions: Male candidates receive more mentions and invitations in newscasts and talk shows and dominate the hard news. In female candidates, gender stereotypes related to their physical appearance or domestic role are still present.
\end{abstract}

KEYWORDS: soft news; hard news; infotainment; electoral campaign; Argentina; gender stereotypes.

\title{
CONTENIDOS
}

1. Introducción. 2. Método. 2.1. Estrategia metodológica. 2.2. Muestra. 3. Resultados. 4. Discusión y conclusiones. 5. Referencias bibliográficas.

\section{Introducción}

La relevancia de la función informativa de los medios de comunicación y, en particular, de la televisión en el ámbito político es una cuestión aceptada por la comunidad científica desde finales del siglo XX (Blumler, 1992; Gerbner, Morgan \& Signorielli, 1994). De la actividad comunicativa se desprende la tesis, también consensuada, de que las noticias emitidas afectan a la percepción que tienen los votantes de la política, lo cual incluye a líderes políticos, partidos y temas (Mazzoleni \& Shulz, 1999; Norris, 2004). Además, en los últimos años, diversos estudios indican que la presencia de candidatos y candidatas en programas de infoentretenimiento político puede influir en las posiciones políticas y el voto de la ciudadanía (Baum, 2005; Brewer y Cao, 2006; Baumgartner y Morris, 2006; Baum y Jamison, 2006).

Una situación parecida se observa acerca de las consecuencias de los estereotipos de género en la percepción que los votantes se forman de las candidatas y la intención de voto hacia ellas. Las investigaciones muestran que cuando los medios usan una retórica sexista y estereotipos de género para referirse a las candidatas, les restan competencia frente al electorado y dañan la percepción que los votantes tienen de ellas en las siguientes dimensiones: confiabilidad, efectividad, fortaleza, experiencia y calificación. Asimismo, la cobertura con estereotipos influye en el descenso la valoración de las candidatas en las encuestas y en la intención de voto hacia ellas (Lake et al., 2010, 2013). 
Esta doble situación vinculada al medio televisivo sobre su influencia en la política y en la repercusión de los estereotipos de género marca este análisis que se centra en estudiar la campaña electoral de las elecciones legislativas de Argentina en 2017, para examinar la cobertura televisiva que candidatos y candidatas recibieron tanto en los programas informativos y en aquellos que realizan infoentretenimiento político o politainment (Nieland, 2008; Sayre \& King, 2010; Schultz, 2012; Berrocal, 2017).

Las preguntas de investigación que plantea el estudio son las siguientes: ¿existen diferencias según el género de las personas que postulan a un cargo de elección popular en la cantidad de menciones e invitaciones que reciben?, ¿hay diferencias en la cantidad de noticias blandas y noticias duras que se comunican según sean hombres o mujeres? Las noticias televisivas referidas a las candidatas, ¿presentan sesgos y estereotipos de género? De ser así, ¿cuáles son esos estereotipos y con qué frecuencia se repiten?

Dichos interrogantes provienen de tres hipótesis a las que responde este trabajo. La primera hipótesis señala que los candidatos reciben más invitaciones que las candidatas para participar en entrevistas, tanto en los programas informativos como en los de infoentretenimiento. La segunda indica que en la cobertura televisiva de las campañas de los candidatos predominan las informaciones duras, mientras que en la de las candidatas las noticias blandas. La tercera afirma que en la cobertura televisiva de las candidatas existen estereotipos de género que, además de recurrentes, son tipificables.

Como se ha referido, una de las preguntas de investigación se enfoca en el análisis cuantitativo de noticias duras y blandas en la cobertura televisiva de candidatas y candidatos durante la campaña electoral. Las soft news o noticias blandas aparecen relacionadas con el nacimiento del infoentretenimiento (García Avilés, 2007) y alude al incremento de noticias de fácil comprensión para el oyente, lector o telespectador, huyendo de las dificultades que pueda presentar el consumo de noticias construidas con numerosas fuentes y datos, así se incluyen historias sensacionalistas, noticias de interés humano o aquellas en las que el foco está en el entretenimiento más que en el contenido serio de la noticia (Thussu, 2007; Jebril et al., 2013 ). Si bien en la literatura científica en comunicación es frecuente encontrar trabajos que hacen referencia a las nociones de soft y hard news, no existe un consenso ni una definición unívoca en el modo en que investigadores y académicos definen esos términos, tanto en sus aspectos conceptuales como en los operacionales. Tuchman $(1972,1973)$ referente clásica en la cuestión, alude a este problema desde una perspectiva periodística. La variable sobre la que originalmente basa la distinción entre ambas categorías de noticias es la "sustancia", entendiendo que las noticias duras necesitan comentarios y análisis; mientras que las blandas involucran escándalos, chismes, farándula e historias de interés humano, con pocas ramificaciones más allá de lo inmediato. Patterson (2000) por su parte afirma que las noticias blandas contienen elementos sensacionalistas relacionados con personalidades famosas, o se refieren a eventos específicos y desconectados de otras historias o tendencias sociales. Lehman-Wilzig y Seletzky $(2010,48)$ reclaman la necesidad de incluir una tercera categoría intermedia de clasificación -a la que proponen denominar "noticias generales"-, definen a las noticias blandas como informaciones "livianas" o "picantes" tales como el cotilleo referido a las celebridades o las denominadas "historias de interés humano"; y a las noticias duras como novedades, hallazgos o descubrimientos del campo de la política, la economía, la sociedad, el medioambiente o la salud pública con ramificaciones e impacto -tanto inmediato como a largo plazo- en el ámbito público, nacional o internacional. Curran et al. (2010) delimitan las noticias duras como aquellas que informan sobre política, administración pública, economía, ciencia, tecnología y temas afines; mientras que las blandas son aquellas que lo hacen acerca de celebridades, cuestiones de interés humano, deportes e historias centradas en el entretenimiento. 
Reinemann et al. (2011, p. 5), tras la realización de un metanálisis de la literatura acerca del tema, identifican cinco dimensiones que, de manera combinada o aislada, se emplean para definir las noticias blandas y duras: 1 . temas/eventos; 2. producción de las noticias; 3. frame; 4. estilo noticioso; 5. recepción de las noticias. Según estos investigadores el $83 \%$ de los estudios usan la dimensión temática para identificar y diferenciar entre noticias blandas y duras, y proponen la siguiente definición:

Cuanto más un ítem noticioso es políticamente relevante, cuanto más se reporte en un modo

temático, se enfoque en las consecuencias sociales de los eventos, sea impersonal y no

emocional en su estilo; más podrá ser considerado como noticia dura. Cuanto más un ítem

noticioso no sea políticamente relevante, cuanto más sea reportado en un modo episódico, se

enfoque en las consecuencias individuales de los eventos, sea personal y emocional en su

estilo; más podrá ser considerado noticia blanda. (Reinemann et al., 2011, p. 13)

La definición de Reinemann et al. (2011) se apoya en tres dimensiones: a la primera, la denominan "dimensión temática", y con ella se proponen distinguir diferentes tipos de relevancia política. Bruns y Marcinowski (1997) entienden el grado de relevancia política como un indicador de la medida en que el contenido de un ítem noticioso se refiere a normas, objetivos, intereses y actividades relacionadas con la preparación, afirmación e implementación de decisiones políticas vinculantes referidas a conflictos sociales. A su vez, la medición de la relevancia política de un ítem noticioso puede realizarse a través de cuatro indicadores empíricos: la mención de actores sociales, de autoridades vinculadas con los procesos de toma de decisión, de un plan o programa propuesto, y de las personas preocupadas por esa decisión (Reinemann et al., 2011). El sentido de la relación estipulada es: a más indicadores presentes, más alto el grado de relevancia política de un ítem noticioso. Los autores entienden que en esta primera dimensión o "dimensión temática", el ablandamiento de una noticia podría significar la reducción de su grado de relevancia política.

Una segunda dimensión es la "dimensión del frame o marco", los autores creen que se debe distinguir entre las informaciones que enfatizan la relevancia o consecuencia pública de un evento, y las noticias que se enmarcan en los aspectos o consecuencias personales o privados. En esta segunda dimensión, el ablandamiento de las noticias estaría indicado por un frame que se centra en cuestiones privadas o personales o en eventos aislados o ejemplares.

Finalmente, la tercera dimensión sobre la cual se asienta la definición, es la "dimensión de estilo", que permite diferenciar entre noticias que incluyen manifestaciones explícitas de las interpretaciones, opiniones e impresiones personales del periodista y las que no incluyen esas perspectivas personales. Adicionalmente, en esta dimensión se debe distinguir si la cobertura informativa incluye elementos verbales o visuales que apele a las emociones o, por el contario, no lo hace. El ablandamiento de las noticias en esta dimensión sería indicativo de un estilo más personal o emocional.

En cuanto al crecimiento de las noticias blandas sobre las duras en el medio televisivo, los estudios arrojan diversas conclusiones. Scott y Gobetz (1992) en su análisis de tres cadenas de televisión nacional en Estados Unidos en el período 1972-1987, concluyen que la cantidad de noticias blandas es pequeña en comparación con la difusión de noticias duras. Waldahl et al. (2009) analizan programas de noticias en los dos principales canales de la televisión de Noruega en 1998, 2000 y 2007, y encuentran que la mayor parte de los contenidos responden a la categoría de las noticias 
duras, sin que el resultado cambiase con el paso del tiempo. En la misma línea se muestra una investigación comparada de carácter transnacional, Curran et al. (2009) concluyen con que existe mayor presencia de noticias duras que blandas en todos los países comprendidos en el estudio. Otras investigaciones, en cambio, indican que las noticias se van tornando mayoritariamente blandas con el transcurrir del tiempo.

La investigación de Patterson (2000), en la que se analiza una muestra de más de cinco mil noticias difundidas por dos cadenas de televisión diferentes en Estados Unidos, proyecta una mayor presencia de noticias sensacionalistas, más centradas en el interés humano, autorreferentes y con carencia de enfoques colectivistas o políticos. Específicamente en el tema de la observación de noticias duras y blandas difundidas por la televisión en un contexto electoral, Donsbach y Bütner (2005) al examinar los informativos de las cuatro cadenas principales de la televisión alemana, durante las cuatro semanas anteriores a las elecciones nacionales en 1983, 1999 y 1998, concluyen que los temas políticos fueron viéndose reducidos de elección a elección en tres de los cuatro informativos, al tiempo que se incrementaban en ellos cuestiones como historias y narrativas dramáticas, cuestiones anecdóticas y se registraba un aumento de las imágenes con fuerte contenido emocional.

El enfoque que se afronta en esta investigación resulta novedoso, ya que no se han localizado antecedes científicos que hayan evaluado la presencia y proporción de información dura y blanda (hard news y soft news) en un contexto electoral y con relación a una variable como el género, tal como lo hace el presente estudio.

Los análisis que sí que se han encontrado pero que difieren del objeto de esta investigación tienen que ver con la relación de la variable "género" y el tipo de secciones y temas noticiosos que se asignan dentro de la propia práctica periodística a los reporteros según sean hombres o mujeres. Así, Cann y Mohr (2001) argumentan que a las mujeres se les solicita la redacción de más noticias blandas en comparación con sus pares hombres y a ellos más noticias duras que a ellas.

Esta investigación propone, por tanto, examinar un área poco explorada: noticias blandas y duras en el ámbito de la política, en el contexto específico de una campaña electoral, y con relación a un área de la actividad humana también definida: hombres y mujeres que se dedican de manera profesional a la actividad política o aspiran a hacerlo.

El interés por incluir la categoría de los estereotipos de género tiene que ver con su importancia, tal y como señalan, numerosos trabajos sobre la cobertura televisiva de candidatas electorales. Los estereotipos de género han sido reconocidos como una de las principales causas para que las mujeres se enfrenten a más obstáculos que los hombres a la hora alcanzar posiciones de liderazgo en general (Molero, 2004) y la política no representa una excepción.

En el ámbito político, existe una marcada desigualdad desfavorable a las mujeres en la ocupación de posiciones de liderazgo. De acuerdo a los datos presentados en el "Mapa de las Mujeres en la Política 2017", elaborado por la Unión Interparlamentaria y ONU Mujeres, sólo 17 mujeres eran jefas de Estado o de gobierno; un $23 \%$ ocupaban escaños en los parlamentos del mundo; el $19 \%$ eran portavoces parlamentarias; en sólo 6 de 186 países tenían el mismo número de ministras y ministros y, únicamente, el $18 \%$ de los ministros de gobierno del mundo eran mujeres.

La tendencia a asociar el liderazgo con el estereotipo de lo masculino aprendida en la socialización temprana tiene consecuencias reales que afectan de manera negativa a las posibilidades de las mujeres para ocupar espacios de poder, liderazgo e influencia política y económica. De ahí la importancia que tienen los medios de comunicación en la creación, reproducción y mantenimiento de 
los estereotipos de género en general, y los referidos a mujeres políticas y candidatas en particular (García Beaudoux, 2017, 2018; García Beaudoux, D’Adamo y Gavensky, 2018).

A continuación, se presentan los resultados del trabajo empírico destinado a establecer los sesgos y estereotipos de género en la cobertura televisiva informativa y de infoentretenimiento de las candidatas y candidatos electorales, en la campaña de las elecciones legislativas de 2017 en Argentina.

\section{Método}

\subsection{Estrategia metodológica}

El método seleccionado es el análisis de contenido para examinar las hard news y soft news localizadas en los programas televisivos que forman la muestra de este trabajo. Dado que el alcance del estudio es descriptivo y se propone realizar una descripción sistemática y cuantitativa, el análisis de contenido se presenta como el método más apropiado para la exploración de las unidades de información que conforman la muestra.

El método de análisis de contenido supone aplicar un procedimiento estandarizado. Es decir, ha de someterse a ciertas reglas, emplear procedimientos que puedan ser utilizados en otras investigaciones y que los resultados obtenidos puedan corroborarse (Krippendorf, 1990, 1997). El estudio, de acuerdo con la definición de Abela (2008), utiliza el análisis de contenido como un conjunto de técnicas tendentes a explicar y sistematizar el contenido de los mensajes comunicativos ya sean textos, sonidos o imágenes con ayuda de indicios cuantificables o no. Todo ello con el objetivo de efectuar deducciones lógicas concernientes a la fuente, al emisor y su contexto o eventualmente a sus efectos. En definitiva, se trata de indagar en los mensajes manifiestos y latentes de un cuerpo de comunicados (Krippendorff, 2013; McQuail, 2013).

Para el registro y la medición se ha diseñado un protocolo de codificación que fue sometido a una prueba piloto en la que participaron los tres codificadores que efectuaron la medición definitiva. Con referencia a la fiabilidad del análisis se siguió el modelo de Lemish y Tidhar (1999), una fórmula basada en el consenso entre los codificadores para elegir una única entrada o categoría para cada variable. En las situaciones en la que los codificadores no lograban llegar a un acuerdo absoluto, se siguió el procedimiento de Garramone, Steele y Pinkleton (1991) y el desacuerdo fue resuelto eligiendo la codificación seleccionada por dos de los tres.

La investigación examina tanto la presencia de "sesgos de género", como de "estereotipos de género" en la cobertura que se realizó de candidatos y candidatas en los programas informativos y de infoentretenimiento televisivo. El concepto "sesgo de género" se utiliza en el sentido propuesto por Moss y Brown (1979) para designar el comportamiento de los periodistas y profesionales de la comunicación al enfatizar los atributos según el género de candidatas y candidatos electorales, aunque dicho énfasis no sea necesario y no se encuentre justificado. La noción de "estereotipo de género", en cambio, alude a la inclusión y refuerzo en la cobertura periodística de creencias socialmente compartidas que describen y prescriben cómo deben ser y actuar hombres y mujeres, los rasgos y comportamientos esperados para cada uno de ellos (García Beaudoux, 2017).

Las categorías examinadas fueron la siguientes: asistencia de candidatas y candidatos a programas informativos y de politainment; menciones en dichos programas a líderes y lideresas políticos; establecimiento cuantitativo de hard news y soft news; diferenciación en menciones, comentarios o 
preguntas a candidatos y candidatas enmarcando dichas alusiones en el frame "blando o duro"; así como el establecimiento de estereotipos de género cuando se alude a la mujer política.

Además, con el objeto de detectar y clasificar los estereotipos de género se ha utilizado una clasificación ya probada, consistente en definir operacionalmente a los estereotipos de género como cualquier sesgo en la cobertura periodística que al referir a las candidatas no mencione sus propuestas políticas, partidos políticos, posicionamiento ideológico, trayectoria política, carrera profesional, experiencia previa, actividad pública o cualquier otro "factor político" y, en cambio, haga énfasis en los aspectos de sus vidas privadas y domésticas, rutinas cotidianas, sus familias, hijos, parejas, situación sentimental, personalidades, estados anímicos y emocionales, gustos, relaciones personales, apariencia física, o cualquier otro "factor extra-político" (García Beaudoux, D’Adamo y Gavensky, 2018). Esta definición de estereotipo de género apunta a un sentido de "noticia blanda" coincidente con la calificación de Reinemann et al. (2011) que se refiere a ella como un ítem noticioso políticamente irrelevante, reportado de modo episódico, con un frame individual y estilo emocional.

\subsection{Muestra}

El periodo de recogida de la muestra es de 31 días y corresponde al espacio temporal establecido entre el 19 de septiembre y el 19 de octubre de 2017, último mes de la campaña electoral, dado que la fecha de la votación fue el 22 de octubre y que la prohibición de hacer campaña se inicia a las cero horas del día 20 de octubre.

El criterio tenido en cuenta para seleccionar la muestra, tanto de los programas informativos como de los de infoentretenimiento televisivo, es su nivel de audiencia. Así, se seleccionaron 6 programas de infoentretenimiento y 4 informativos. Se decidió esta distribución porque mientras algunos programas de infoentretenimiento se emiten sólo los sábados o domingos, la mayoría de los informativos se emiten cuatro o cinco veces a la semana. La muestra total está conformada por 132 emisiones, 61 de ellas corresponden a programas de infoentretenimiento, lo que representa el $46 \%$ de los programas analizados; mientras que 71 a informativos televisivos, lo que representa el $54 \%$ de los programas analizados. Los programas de infoentretenimiento televisivo que conforman la muestra son los siguientes. Por Canal 13, "El diario de Mariana" (2 horas de duración cada emisión), "La noche de Mirtha" ( 2 horas de duración cada emisión) y "Almorzando con Mirtha" (2 horas de duración cada emisión). Por Telefe, "Podemos Hablar" (3 horas de duración) y por América, "Polémica en el Bar (1 hora de duración)". Los programas informativos, se distribuyen de la siguiente manera: "Telenueve Central" (una hora de duración) que es el telediario de Canal Nueve e "Intratables" (2 horas de duración) y Animales Sueltos" del canal América (2 horas de duración). El programa "Periodismo para Todos" (dos horas de duración), emitido por Canal 13, se analizó para ambas categorías, diferenciado según el siguiente criterio de clasificación: el monólogo con el que su conductor inicia el programa cada domingo y la parodia humorística de la política nacional argentina denominada "House of Grieta" (media hora de duración entre ambos segmentos), que se emite tras el monólogo, se categorizaron como infoentretenimiento; mientras que los informes de investigación periodística de la realidad política que se emiten en el resto del programa, se clasificaron como programa político informativo (una hora y media de duración). Cada una de las 132 emisiones que conforman la muestra se consideró una unidad de análisis.

Con referencia a la muestra de candidatas y candidatos sobre los que se analizó la cobertura televisiva recibida, está conformada por los diputados y senadores nacionales para la elección de octubre de 2017, que ocupaban los dos primeros lugares en las tres listas electorales que resultaron más votadas en los dos distritos electorales analizados (CABA -Ciudad Autónoma de Buenos Aires- 
y Provincia de Buenos Aires, dos de los principales distritos electorales de Argentina) en las PASO (Primarias Abiertas Simultáneas y Obligatorias) el 13 de agosto de 2017. La muestra total es de 18 candidatos y candidatas, y la submuestra por género es de 10 candidatas y 8 candidatos. Cuatro mujeres encabezaban listas, mientras que cinco varones eran cabeza de las mismas.

De la fuerza política Cambiemos se analizó la cobertura recibida por Esteban Bullrich y Gladys González como candidatos para el senado por la Provincia de Buenos Aires, Graciela Ocaña y Héctor Toty Flores para la Cámara de Diputados por la Provincia de Buenos Aires; y Elisa Carrió y Carmen Polledo como candidatas a diputadas nacionales por CABA. De la fuerza política Unidad Ciudadana se analizó la cobertura de Cristina Fernández de Kirchner y Héctor Taiana como candidatos a senadores nacionales por la Provincia de Buenos Aires, de Fernanda Vallejos y Roberto Salvarezza como candidatos a diputados nacionales por ese mismo distrito, y de Daniel Filmus y Gabriela Cerrutti como candidatos a diputados nacionales por CABA. Por la fuerza política 1 País, se analizó la cobertura de Sergio Massa y Margarita Stolbizer candidatos a senadores nacionales por la Provincia de Buenos Aires, y de Felipe Solá y Mirta Tundis candidatos a diputados por ese mismo distrito. En CABA, la tercera fuerza resultante de las PASO no fue 1 País sino Evolución Ciudadana, y se analizó la cobertura recibida por sus candidatos a diputados nacionales, Martín Lousteau y Carla Carrizo.

\section{Resultados}

Los resultados obtenidos se presentan a continuación, diferenciados de acuerdo a las categorías "programas de infoentretenimiento político televisivo" y "programas informativos televisivos". La primera categoría refiere a los programas televisivos que combinan la información con el entretenimiento; y la mayoría de ellos responden a tres formatos posibles: 1) informativos espectacularizados; 2) magazines o "programas contenedores" que incluyen la política en sus emisiones, bien en tertulias, bien en entrevistas o en informaciones con un carácter frívolo o superficial; 3) programas de parodia informativa/política o infoshow (Berrocal y Campos, 2012).

En cuanto a la frecuencia de aparición, la presencia total de los candidatos en las emisiones de los programas televisivos analizados en conjunto, triplicó a la de las candidatas (tabla 1). Candidatas y candidatos estuvieron personalmente presentes en 36 del total de 132 programas de televisión analizados durante el período seleccionado, lo que constituye el $27 \%$ de la muestra. El $25 \%$ de esas veces que asistieron, lo hicieron a programas de infoentretenimiento televisivo y el $75 \%$ a programas de tipo informativo. Considerando ambos tipos de programas en conjunto, las candidatas representaron el 30\% y los candidatos el $70 \%$ de los entrevistados que se vieron en televisión durante la campaña. Si se consideran los programas por tipo, la presencia de ellas se concentró el 10\% de las veces (una única oportunidad) en programas de infoentretenimiento y el $90 \%$ en los informativos, mientras que en el caso de los candidatos fue $32 \%$ en el primer tipo de programas y $68 \%$ en el segundo.

Tabla 1. Asistencia de candidatos y candidatas a programas informativos y de infoentretenimiento.

\begin{tabular}{|l|l|l|}
$\begin{array}{l}\text { Candidatas y candidatos asistieron } \\
\text { 36 programas de TV, lo que } \\
\text { representa el } 27 \% \text { de la muestra } \\
\text { analizada }\end{array}$ & Candidatas & Candidatos \\
\hline $\begin{array}{l}\text { Asistencia a programas de } \\
\text { infoentretenimiento }\end{array}$ & $\begin{array}{l}\text { 1 programa, que representa } \\
\text { el } 10 \% \text { de la presencia total } \\
\text { de las candidatas en } \\
\text { programas de TV }\end{array}$ & $\begin{array}{l}10 \text { programas, que representan el } \\
32 \% \text { de la presencia total de los } \\
\text { candidatos en programas de TV }\end{array}$ \\
\hline
\end{tabular}


RLCS, Revista Latina de Comunicación Social, 77, 275-293

[Investigación] DOI: 10.4185/RLCS-2020-1458 | ISSN 1138-5820 | Año 2020

\begin{tabular}{|l|l}
\hline Asistencia a programas & 8 programas, que \\
informativos & representan el 90\% de la \\
presencia total de las \\
candidatas en programas de \\
TV
\end{tabular}

17 programas, que representan el $68 \%$ de la presencia total de los candidatos en programas de TV

Fuente: elaboración propia.

Con independencia de la asistencia o presencia física de candidatas y candidatos en los programas tanto informativos como de infoentretenimiento, detallada en la tabla 1, candidatas y candidatos fueron mencionados un total de 430 veces durante el período seleccionado (tabla 2). Las menciones se identificaron en la totalidad de las 132 emisiones de programas analizadas.

Con referencia a las menciones totales, el $32 \%$ sucedieron en programas de infoentretenimiento televisivo y el $68 \%$ en programas de tipo informativo. Cabe destacar que una única candidata, la ex presidenta Cristina Fernández de Kirchner, concentró ella sola más del 79\% de las menciones a candidatas mujeres que se hicieron en la totalidad de los programas de televisión analizados. Si se consideran los programas por tipo, las menciones a ellas sucedieron el $38 \%$ de las veces en programas de infoentretenimiento y el $62 \%$ en los informativos, mientras que los candidatos obtuvieron el $19 \%$ de las menciones en el primer tipo de programas y el $81 \%$ en el segundo.

Tabla 2. Menciones de candidatas y candidatos en programas informativos y de infoentretenimiento.

\begin{tabular}{|l|l|l|}
\hline Candidatas & $\begin{array}{l}\text { Menciones en programas de } \\
\text { infoentretenimiento }\end{array}$ & $\begin{array}{l}\text { Menciones en programas } \\
\text { informativos }\end{array}$ \\
\hline & $\begin{array}{l}111 \text { menciones, que representan } \\
\text { el 38\% de las menciones del } \\
\text { total de la muestra }\end{array}$ & $\begin{array}{l}\text { 185 menciones, que representan } \\
\text { el 62\% de las menciones del } \\
\text { total de la muestra }\end{array}$ \\
& $\begin{array}{l}\text { En 102 de ellas la candidata } \\
\text { mencionada fue Cristina } \\
\text { Fernández Kichner y solo 9 } \\
\text { veces otras candidatas }\end{array}$ & $\begin{array}{l}\text { En 133 de ellas la candidata } \\
\text { mencionada fue Cristina } \\
\text { Fernández Kichner y 52 veces } \\
\text { otras candidatas }\end{array}$ \\
\hline \multirow{2}{*}{ Candidatos } & $\begin{array}{l}\text { 26 menciones, que representan el } \\
19 \% \text { de las menciones del total } \\
\text { de la muestra }\end{array}$ & $\begin{array}{l}\text { 10 menciones, que representan el } \\
81 \% \text { de las menciones del total } \\
\text { de la muestra }\end{array}$ \\
\hline
\end{tabular}

Fuente: elaboración propia.

Con el objetivo de indagar si existen diferencias estadísticamente significativas en el promedio de menciones en los programas de infoentretenimiento e informativos según el género del candidato, se realizó una prueba t de student arrojando diferencias a favor de las mujeres (infoentreteniemiento $\mathrm{t}=.1,34 ; \mathrm{p}=.00$, informativo $\mathrm{t}=2,61 ; \mathrm{p}=.00$ ). Es decir, las candidatas fueron más mencionadas en ambas clases de programas, lo que se explica sobre todo porque una de ellas, Cristina Fernández, por su calidad de reciente ex presidenta de la nación, concentró la atención mediática. Sin embargo, al realizar la comparación según el tipo de programa, las diferencias estadísticamente significativas son a favor de los programas de tipo infoentretenimiento para las candidatas $(t=1,39 ; \mathrm{p}=.00)$ y de $\operatorname{los}$ programas de tipo informativo para los candidatos $(\mathrm{t}=1,03 ; \mathrm{p}=.00)$.

De las menciones, comentarios y preguntas que candidatas y candidatos recibieron en programas de infoentretenimiento televisivo, el 53\% fue información blanda y el 47\% dura (tabla 3). En el caso de las mujeres, las menciones, comentarios y preguntas que se les formularon fueron en el $68 \%$ de las 
veces contenido blando y solo $32 \%$ duro. Al analizar la situación de los hombres en la misma dimensión, la perspectiva se invierte: el $42 \%$ fueron menciones, comentarios y preguntas de carácter blando, mientras que el $58 \%$ fue de carácter duro. Además, en los casos en los que se comunicó información dura el 19\% de las veces se discutieron las propuestas de los candidatos, mientras que en el caso de las candidatas eso sólo sucedió en el 9\% de las ocasiones.

Las categorías de contenido detalladas en las tablas 3, 4 y 5, se establecieron mediante un proceso inductivo, es decir, se crearon a medida que se manifestaban en el corpus analizado. Dicha estrategia metodológica se encuentra legitimada en que el análisis de contenido es

el conjunto de técnicas de análisis de las comunicaciones tendentes a obtener indicadores

(cuantitativos o no) por procedimientos sistemáticos y objetivos de descripción del contenido

de los mensajes permitiendo la inferencia de conocimientos relativos a las condiciones de

producción/recepción (contexto social) de estos mensajes. (Bardin, 1996, p. 32)

Tabla 3. Soft news y hard news en menciones, comentarios y preguntas a candidatos y candidatas en programas de infoentretenimiento.

\begin{tabular}{|c|c|c|c|}
\hline Tipo de información & Contenido específico & Candidatas & Candidatos \\
\hline \multirow[t]{8}{*}{ Información blanda (soft news) } & Total & $68 \%$ & $42 \%$ \\
\hline & $\begin{array}{l}\text { Aspectos de sus vidas } \\
\text { privadas }\end{array}$ & $17 \%$ & $31 \%$ \\
\hline & Vestimenta o apariencia física & $16 \%$ & $7 \%$ \\
\hline & $\begin{array}{l}\text { Anécdotas (personales o de } \\
\text { camnaña) }\end{array}$ & $23 \%$ & $32 \%$ \\
\hline & Sentimientos personales & $16 \%$ & $17 \%$ \\
\hline & $\begin{array}{l}\text { Ridiculizaciones, ingredientes } \\
\text { cómicos }\end{array}$ & $16 \%$ & $10 \%$ \\
\hline & Deportes & $0 \%$ & $3 \%$ \\
\hline & Otros & $12 \%$ & $0 \%$ \\
\hline \multirow[t]{7}{*}{ Información dura (hard news) } & Total & $32 \%$ & $\mathbf{5 8 \%}$ \\
\hline & Presentación de propuestas & $9 \%$ & $19 \%$ \\
\hline & $\begin{array}{l}\text { Presentación/ discusión de } \\
\text { datos }\end{array}$ & $0 \%$ & $17 \%$ \\
\hline & Debate de sus posiciones & $0 \%$ & $20 \%$ \\
\hline & Denuncias corrupción & $49 \%$ & $9 \%$ \\
\hline & Situación encuestas electorales & $21 \%$ & $12 \%$ \\
\hline & $\begin{array}{l}\text { Realización de un debate } \\
\text { electoral }\end{array}$ & $13 \%$ & $8 \%$ \\
\hline
\end{tabular}


RLCS, Revista Latina de Comunicación Social, 77, 275-293

[Investigación] DOI: 10.4185/RLCS-2020-1458 | ISSN 1138-5820 | Año 2020

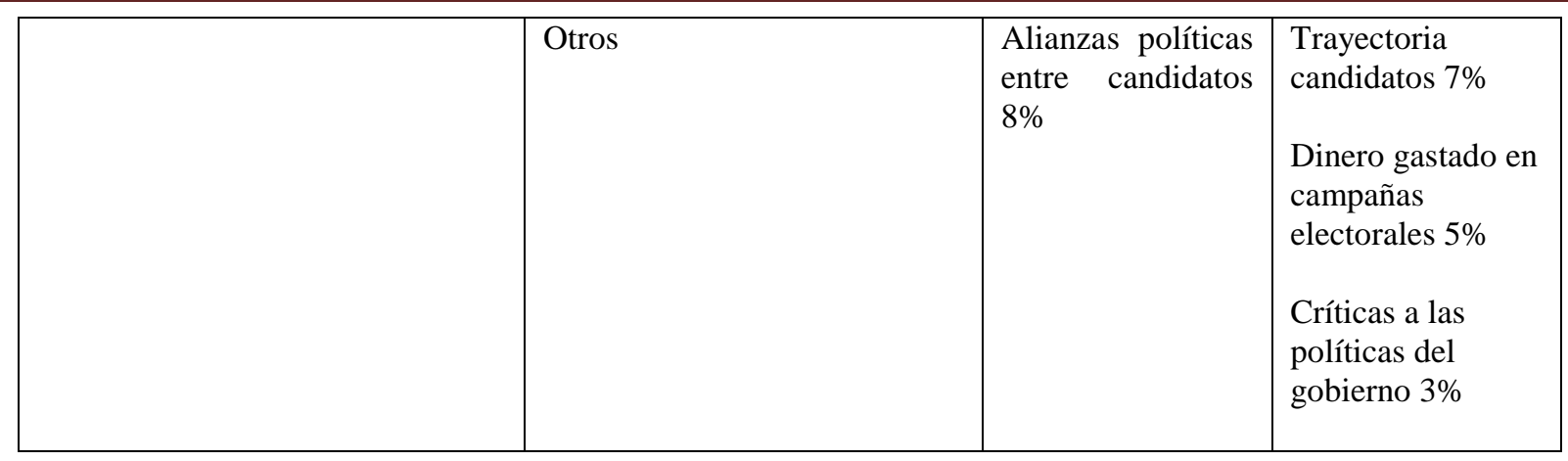

Fuente: elaboración propia.

Con el objetivo de indagar si existen diferencias estadísticamente significativas en el tipo de información (blanda o dura) según el género de las personas candidatas, en los programas de infoentretenimento, se realizó una prueba t de student que arrojó diferencias a favor de las candidatas $(\mathrm{t}=3,28 ; \mathrm{p}=.05)$ para la información blanda, $\mathrm{y}$ en el caso de los candidatos para la información dura $(\mathrm{t}=2,80 ; \mathrm{p}=.05)$. En otras palabras, en las candidatas se registra de manera significativa contenido de información más soft o blanda; y en los candidatos, también de manera significativa, contenido de información más hard o dura.

En lo que refiere a las menciones, comentarios y preguntas en los programas informativos de televisión, en el caso de las candidatas el 30\% de las veces fueron de contenido blando y el $70 \%$ duro, mientras que en el de los candidatos el $21 \%$ fueron de carácter blando y el $79 \%$ de carácter duro (tabla 4). El 22\% de la información dura relacionada con los candidatos se concentró en sus propuestas de campaña, mientras que eso solamente sucedió un $6 \%$ de las veces con las candidatas.

Tabla 4. Soft news y hard news en menciones, comentarios y preguntas en programas informativos.

\begin{tabular}{|c|c|c|c|}
\hline Tipo de información & Contenido específico & Candidatas & Candidatos \\
\hline \multirow{8}{*}{$\begin{array}{l}\text { Información blanda (soft } \\
\text { news) }\end{array}$} & Total & $30 \%$ & $21 \%$ \\
\hline & Aspectos de sus vidas privadas & $12 \%$ & $28 \%$ \\
\hline & Vestimenta o apariencia física & $17 \%$ & --- \\
\hline & Anécdotas (personales o de campaña) & $35 \%$ & $45 \%$ \\
\hline & Sentimientos personales & $20 \%$ & $14 \%$ \\
\hline & Ingredientes cómicos & $3 \%$ & $9 \%$ \\
\hline & Hobbies/Aficiones & --- & $2 \%$ \\
\hline & Otros & $13 \%$ & $2 \%$ \\
\hline \multirow{5}{*}{$\begin{array}{l}\text { Información dura (hard } \\
\text { news) }\end{array}$} & Total & $70 \%$ & $79 \%$ \\
\hline & Presentación propuestas & $6 \%$ & $22 \%$ \\
\hline & Discusión de datos & $1 \%$ & $3 \%$ \\
\hline & Debate ideológico & $9 \%$ & $14 \%$ \\
\hline & Situación encuestas electorales & $25 \%$ & $14 \%$ \\
\hline
\end{tabular}


RLCS, Revista Latina de Comunicación Social, 77, 275-293

[Investigación] DOI: 10.4185/RLCS-2020-1458 | ISSN 1138-5820 | Año 2020

\begin{tabular}{|l|l|l|l|}
\hline \hline & & & \\
\hline & $\begin{array}{l}\text { Denuncias contra la ex presidenta Cristina } \\
\text { Fernández }\end{array}$ & $19 \%$ & $17 \%$ \\
\hline & Realización de un debate electoral & $15 \%$ & $10 \%$ \\
\hline & Actos de campaña & $14 \%$ & $8 \%$ \\
\hline & Lanzamientos spots electorales televisivos & $11 \%$ & $7 \%$ \\
\cline { 2 - 4 } & Otros & & $\begin{array}{l}\text { Obras públicas } \\
5 \%\end{array}$ \\
\hline
\end{tabular}

Fuente: elaboración propia.

Con el objetivo de indagar si existen diferencias estadísticamente significativas en el tipo de información (blanda o dura) según el género de las personas candidatas en los programas informativos, se realizó una prueba t de student que arrojó, al igual que en el caso de los programas de infoentretenimiento, diferencias a favor de las candidatas $(\mathrm{t}=6,46 ; \mathrm{p}=.00)$ para la información blanda, $y$ en el caso de los candidatos en el tipo de información dura $(t=8,04 ; p=.00)$. Eso significa que la cobertura y el tratamiento de las candidatas en los medios incluye de manera significativa contenido de información más blanda, y en los candidatos, también de manera significativa, contenido de información más dura.

Finalmente, en lo que respecta al refuerzo de estereotipos de género, la presencia porcentual es baja, las tipificaciones muestran una escasa dispersión y se concentran en unos pocos sesgos y estereotipos (tabla 5). En ambas clases de programas la mayor frecuencia de sesgos se concentra en torno a la apariencia física de las candidatas. En el caso de los programas de infoentretenimiento, a lo anterior se suman comentarios acerca de los roles y tareas de cuidado que las candidatas desempeñan en el ámbito de sus vidas domésticas y privadas.

Tabla 5. Estereotipos de género en las menciones a candidatas en programas informativos y de infoentretenimiento.

\begin{tabular}{|l|l|l|}
\hline & $\begin{array}{l}\text { Programas de } \\
\text { infoentretenimiento }\end{array}$ & Programas informativos \\
\hline Estereotipos de género & $11 \%$ & $2 \%$ \\
\hline $\begin{array}{l}\text { Roles de madres/abuelas/ } \\
\text { esposas/novias/cuidadoras de los } \\
\text { demás }\end{array}$ & $1 \%$ & $0 \%$ \\
\hline $\begin{array}{l}\text { Compatibilidad vida doméstica con } \\
\text { la función política }\end{array}$ & $3 \%$ & $0 \%$ \\
\hline $\begin{array}{l}\text { Temperamento autoritario o } \\
\text { mandón }\end{array}$ & $12 \%$ & $11 \%$ \\
\hline Vestimenta y apariencia física & $5 \%$ & $3 \%$ \\
\hline Inestabilidad emocional & $7 \%$ & $2 \%$ \\
\hline $\begin{array}{l}\text { Frialdad emocional/ausencia de } \\
\text { manifestación de emociones }\end{array}$ & & \\
\hline
\end{tabular}

Fuente: elaboración propia. 


\section{Conclusiones}

La investigación planteada atiende a tres presunciones de investigación que se han visto corroboradas. La primera hipótesis se valida ya que los candidatos reciben significativamente más invitaciones que las candidatas para participar en entrevistas, tanto en los programas informativos como en los de infoentretenimiento. Ellas recibieron tres veces menos invitaciones que ellos. Tanto candidatos como candidatas recibieron más menciones en los programas de tipo informativo, aunque cabe destacar que ellas obtuvieron muchas más menciones que ellos en los programas de infoentretenimiento televisivo: las alusiones a ellas representan el $38 \%$, mientras que las de ellos el $19 \%$.

De manera parcial se verifica la segunda hipótesis. La presunción inicial esperaba encontrar predominio de noticias duras en la cobertura de los candidatos, mientras que de noticias blandas en la de las candidatas, y esta afirmación sólo es parcialmente correcta. Los hallazgos indican que, con respecto a los hombres predominan las noticias duras tanto en los programas de infoentretenimiento (58\% duras frente a un $42 \%$ blandas) como en los informativos ( $79 \%$ de noticias duras frente a un $21 \%$ de noticias blandas). Mientras que, cuando se trata de las candidatas, en los programas de infoentretenimiento predominan las noticias blandas de ellas (68\% blandas frente a $32 \%$ duras) pero en los programas informativos, al igual que ocurre con los hombres, hay más noticias duras asociadas con sus nombres (70\% duras frente a $30 \%$ blandas), aunque cabe matizar que en una proporción inferior a la de los hombres (70\% de noticias duras para ellas, $79 \%$ para ellos).

En lo que respecta a la tercera hipótesis, esto es, que en la cobertura televisiva de las candidatas existirían estereotipos de género que además de recurrentes, serían tipificables; los resultados indican que los estereotipos existen y que se concentran en los tipos estables, predominando en el caso específico de esta campaña los comentarios sesgados hacia la apariencia física de las candidatas y los comentarios acerca de sus roles domésticos vinculados con tareas de cuidado.

La mayor presencia de mujeres en programas de infoentretenimiento televisivo explica, en parte, por qué son también sujeto de mayor cantidad de noticias blandas, dadas las características propias de esos programas. Asimismo, la mayor presencia en ese tipo de programas explica por qué dentro del espectro de tópicos que ofrecen las noticias blandas, las cuestiones que más se han destacado de las candidatas son las vinculadas con su apariencia física y las tareas del hogar. La dinámica psicosocial que se establece entre los estereotipos y los sesgos de género, indica que los primeros activan a los segundos. Es decir, los estereotipos predominantes socialmente compartidos, facilitan la aparición de sesgos de género al momento de las entrevistas periodísticas, lo que se encontraría aún más acentuado cuando tienen lugar en programas de infoentretenimiento. El riesgo de reproducir sesgos y naturalizar estereotipos en la cobertura periodística de las campañas electorales es, siguiendo la línea de pensamiento de Kahneman (2012), que ellos funcionen como atajos o heurísticos cognitivos tanto en el momento de procesar la información, como de juzgar a las candidatas, así como de tomar la decisión electoral de votarlas o no.

La tendencia a la desigualdad y la cobertura sesgada en términos de género en los medios de comunicación continúa vigente (Trappel, 2019). En el caso de Argentina, Mitchelstein, Andelsman y Boczkowski, (2019), tras analizar más de tres mil artículos periodísticos de los principales medios digitales de dicho país, encuentran que los hombres tienen el doble de probabilidades en comparación con las mujeres de ser citados como fuentes de información o de la noticia; sobre todo en los artículos referidos a política, economía, asuntos públicos, ciencia y tecnología. El problema no se limita a la ausencia de mujeres en calidad de fuentes, sino que, en coincidencia con los hallazgos de esta investigación, otras y otros autores detectan que los frames que utilizan los medios en la 
RLCS, Revista Latina de Comunicación Social, 77, 275-293

[Investigación] DOI: 10.4185/RLCS-2020-1458 | ISSN 1138-5820 | Año 2020

cobertura noticiosa de las mujeres políticas refuerzan estereotipos de género (Fernández, 2014; Baxter, 2017). En la contienda electoral del año 2008 en Estados Unidos, tanto Hillary Clinton como Sarah Palin recibieron preguntas acerca de sus habilidades como madres, y comentarios acerca de la utilización egoísta que habrían realizado de sus propios hijos para promocionar sus ambiciones políticas (Carlin y Winfrey, 2009). De acuerdo a Quevedo y Berrocal (2018), en la medida en que los escenarios de la política se tornan más paritarios, es esperable que los medios de comunicación sumen nuevos marcos interpretativos y estereotipos. El estudio longitudinal realizado por Wagner, Trimble y Sampert (2019) sobre la cobertura que recibieron en los medios de comunicación 10 mujeres y 17 hombres aspirantes a liderar partidos políticos en Canadá desde 1975 hasta nuestros días, apunta en este mismo sentido: las mujeres candidatas recibieron coberturas con sesgos de género, y el discurso con sesgos de género acerca del liderazgo político ha cambiado poco con el paso del tiempo. Waters y colegas (2019), a partir de su estudio acerca de mujeres candidatas durante una campaña electoral en el Estado de Texas, señalan que la cobertura acerca de las mujeres políticas refuerza estereotipos de género de lo que se considera "femenino", y que ello encierra un peligro para ellas: las candidatas que no son percibidas como femeninas - por ejemplo, porque tienden a decir abiertamente lo que piensan, o porque manifiestan públicamente su ambición- corren el riesgo de ser objeto de severas críticas, dado que desafían la prescripción del rol de género. Los resultados expuestos apuntan en el mismo sentido que los hallazgos arrojados por la investigación llevada a cabo por Winfrey y Schnoebelen (2019), en la que se evidencia que los estereotipos de género aún prevalecen en la cobertura de las campañas electorales.

El trabajo realizado apunta a la necesidad de ampliar la investigación considerando otros medios y plataformas de comunicación, a fin de continuar observando y registrando de manera sistemática el tipo de información dominante, los sesgos de género y la presencia de estereotipos de las candidatas durante las campañas electorales.

\section{Referencias bibliográficas}

Abela, J. A. (2005). Las técnicas de análisis de contenido: Una revisión actualizada. Universidad de Granada.

Bardin, L. (2011). Análises de Conteúdo. Almedina Brasil.

Baum M. A. (2005). Talking the Vote: Why Presidential Candidates Hit the Talk Show Circuit. American Journal of Political Science, 49(2), 213-234.

Baum, M. A. \& Jamison, A. S. (2006). The Oprah effect: Howsoft news helps inattentive citizens vote consistently. Journal of Politics, 68(4), 946-959.

Baumgartner, J. \& Morris, J. (2006). The Daily Show Effect. Candidate Evaluations, Efficacy and American Youth. American Politics Research, 34(3), 341-367.

Baxter, J. (2017). Women Leaders and Gender Stereotyping in the UK Press: A Post-structuralist Approach. Palgrave Macmillan.

Berelson, B. (1952). Content Analysis in Communication Researches. Free Press.

Berrocal G. (Coord.) (2017). Politainment. La política espectáculo en los medios de comunicación. Tirant Lo Blanch. 
Berrocal G. \& Campos Domínguez, E. (2012). El infoentretenimiento político en Internet. Un análisis de los vídeos más vistos en YouTube de José Luis Rodríguez Zapatero y Mariano Rajoy, en S. Berrocal Gonzalo y E. Campos Domínguez (Coords), La investigación en periodismo político en el entorno de los nuevos medios, (pp. 75-90). Sociedad Española de Periodística.

Blumler, J. G. (Ed.) (1992). Television and the Public Interest. Sage.

Brewer, P. R. \& Cao, X. (2006). Candidate Appearences on Soft News Shows and Public Knowledge About Primary Campaigns. Journal of Broadcasting and Electronic Media, 50(1), 18-35.

Bruns, T. \& Marcinowski; F. (1997). Politische Information im Fernsehen: Eine Längsschnittstudie zur Veránderung der Politikvermittlung in Nachritchen uns politischen Informationssendugen. Leske + Bludrich.

Cann, D. J. \& Mohr, P. B. (2001). Journalists and Source Gender in Australian Television News. Journal of Broadcasting \& Electronic Media, 45, 162-174.

Carlin, D. \& Winfrey, K. (2009). Have You Come a Long Way, Baby? Hillary Clinton, Sarah Palin and Sexism in 2008 Campaign Coverage. Communication Studies, 60(4), 326-43.

Curran, J. \& Iyengar, S. \& Lund, A. B. \& Salovaara-Moring, I. (2009). Media system, public knowledge and democracy: A comparative Study. European Journal of Communication, 24(1), 526.

Curran, J. \& Salovaara-Moring, I. \& Cohen, S. \& Iyengar, S. (2010). Crime, foreigners and hard news: A cross-national comparison of reporting and public perception. Journalism, 11(1), 3-19.

Donsbach, W. \& Búttner, K. (2005). Boulevardisiertungstrend in deutschen Fernséhnachrichten. Publizistic, 50(1), 21-38.

Fernández, N. (2014). Medios, género y política. Representación en prensa escrita de las ministras españolas (1982-2011). (Tesis doctoral). Universidad Autónoma de Barcelona, Barcelona, España.

García Avilés, J. A. (2007). El infoentretenimiento en los informativos líderes de audiencia en la Unión Europea. Anàlisi, 35, 47-63.

García Beaudoux, V. (2014. Influencia de la televisión en la creación de estereotipos de género y en la percepción social del liderazgo femenino. La importancia de la táctica de reencuadre para el cambio social. Ciencia Política, 9(18), 47-66.

García Beaudoux, V. (2017). ¿Quién teme el poder de las mujeres? Bailar hacia atrás con tacones altos. Grupo 5.

García Beaudoux, V. (2018). Medios de comunicación, estereotipos de género y liderazgo femenino en América Latina, en F. Freidenberg, M. Caminotti, B. Muñoz-Pogossian y T. Dosek (eds.): Mujeres en la Política: Experiencias nacionales y subnacionales en América Latina. Cap. 4. Instituto Electoral de la Ciudad de México e Instituto de Investigaciones Jurídicas UNAM. 
García Beaudoux, V. \& D’Adamo, O. \& Gavensky, M. (2018). Una tipología de los sesgos y estereotipos de género en la cobertura periodística de las mujeres candidatas. Revista Mexicana de Opinión Pública, (24), 113-129.

Garramone, G. M. \& Steele, M. E. \& Pinkleton, B. (1991). The role of cognitive schemata in determining candidate características effects, en F. Biocca (Ed.), Television and Political Advertising, Vol. 1. Psychological Processes, (pp. 311-328). Erlbaum.

Gerbner, G., Morgan, M. \& Signiorelli, N. (1994). Growing Up with Television: The Cultivation Perspective, en J. Bryant \& D. Zillman. Media Effects. Advances in Theory and Research, (pp. 1742). Lawrence Erlbaum Associates.

Jebril, N., Albaek, E. \& De Vreese, C. H. (2013). Infotainment, Cynism and Democracy: The effects of privatization vs personalization in the news. European Journal of Communication, 28(2), 150-121.

Kahneman, D. (2012). Thinking Fast and Slow. Penguin Random House.

Krippendorff, K. (1990). Metodología del análisis de contenido. Teoría y práctica. Paidós.

Krippendorff, K. (2013). Content analysis: an introduction to its methodology. Sage.

Lake, C. \& Snell, A. \& Gormley, C \& Lethbridge-Cejku, F. (2010). A Simulation of the Impact of Sexism in Campaigns [en linea], Lake Research Partners, Berkeley, 2010. http://www.lakeresearch.com/news/NameIt-ChangeIt/NameItChangeIt.pres.pdf

Lake, C. \& Snell, A. \& Gormley, C. \& Lethbridge-Cejku, F. (2013). An Examination of the Impact of Media Coverage of Womens' Candidates Appearance. Findings of an Online Dial Survey of 1500 Likely Voters Nationwide with an Oversample of 100 Young Women Voters [en linea], Lake Research Partners, Berkeley, 2013. http://wmc.3cdn.net/60f76f6e9c8192224c_34m6b9yo2.pdf

Lehman-Wilzig, S. \& Seletzky, M. (2010). Hard news, soft news, 'general' news: The necessity and utility of an intermediate classification. Journalism, 11(1), 37-56.

Lemish, D. \& Tidhar, C. E. (1999). Still marginal: Women in Israel's television election campaign. Sex roles: A Journal of Research, (41), 389-412.

Mazzoleni, G. \& Schulz, W. (1999). Mediatization of Politics: A Challenge for Democracy? Political Communication, (16), 247-261.

McQuail, D. (2013). Teorias da comunicação de massa. Penso.

Mitchelstein, E., Andelsman, V. \& Boczkowski, P. (2019). Joanne Public vs. Joe Public: News Sourcing and Gender Imbalance on Argentine Digital Media. Digital Journalism, 1-17.

Molero, F. (2004). El liderazgo, en C. Huici Casal y J. F. Morales Domínguez (Dirs.) (2004), Psicología dr Grupos I. Estructura y Procesos, (Cap. 5, pp. 141-170), Universidad Nacional de Educación a Distancia.

Nieland, J. (2008). Politainment. En W. Donsbach, The International Encyclopedia of Communication. Blackwell Publishing. 
Norris, P. (2004). A Virtuous Circle: Political Communications in Postindustrial Societies. Harvard University.

Patterson, T. E. (2000). Doing Well and Doing Good: How Soft News Are Shrinking the News Audience and Weakening Democracy. Harvard University Press.

Quevedo Redondo, R. y Berrocal Gonzalo, S. (2018). Frames, estereotipos y mujeres políticas. Más poder local, (36), 32-36.

Reinemann, C. \& Stanyer, J. \& Scherr, S. \& Legnante, G. (2011). Hard and soft news: A review of concepts, operationalizations and key findings. Journalism, 13(2), 1-19.

Sayre, S. \& King, C. (2010). Entertetainment and society. Influences, impacts and innovations. Routledge.

Schultz, D. (2012). Politainment: The ten rules of contemporary politics: a citizens' guide to understanding campaigns and elections. Amazon.com.

Tchuman, G. (1972). Objectivity as a Strategic Ritual. American Journal of Sociology, 77, 660-679.

Scott, D. K., \& Gobetz, R.H. (1992). Hards news/soft news content of the national broadcast networks. Journalism-Quarterly, 1972-1987, 69(2), 406-412.

Thussu, D. K. (2007). News as Entertainment: The Rise of Global Infotainment. Sage.

Trappel, J. (2019). Inequality, (New) Media and Communications, en J. Trappel (Ed.), Digital Media Inequalities: Politics against Divides, Distrust, and Discrimination, (pp. 9-30). Nordicom.

Tuchuman, G. (1972). Objectivity as a Strategic Ritual. American Journal of Sociology, 77, 660-679.

Tuchman, G. (1973). Making news by doing work: Routinizing the unexpected. The American Journal of Sociology, 7(1), 387-402.

Unión Interparlamentaria y ONU Mujeres (2017). Women in politics 2017 map. UN Women. http://www.unwomen.org/en/digital-library/publications/2017/4/women-in-politics-2017-map

Wagner, A., Trimble, L. y Sampert, S. (2019). One Smart Politician: Gendered Media Discourses of Political Leadership in Canada. Canadian Journal of Political Science/Revue canadienne de science politique, 52(1), 141-162.

Waldahl, R. \& Andersen, M. B. \& Ronning, H. (2009). TV-nyheteness verden. Universitetsforlaget.

Waters, S. E., Dudash-Buskirk, E. A. \& Pipan, R. M. (2018). Battleground Texas: Gendered Media Framing of the 2014 Texas Gubernatorial Race. Journal of Feminist Scholarship, 14(14), 55-71.

Winfrey, K. L. \& Schnoebelen, J. M. (2019). Running as a woman (or man): a review of research on political communicators and gender stereotypes. Review of Communication Research, 7, 109-138. 


\section{AUTORES:}

\section{Virginia García Beaudoux}

Doctora en Psicología. Profesora titular de Campañas Electorales, Propaganda y Opinión Pública en la Universidad de Buenos Aires. Codirectora del Centro de Opinión Pública de la Universidad de Belgrano. Investigadora de CONICET. Es profesora en diversos programas de comunicación política: en el ICPS de la Universidad Autónoma de Barcelona; el Instituto Universitario Ortega y Gasset, y el Máster en Asesoramiento de Imagen y Consultoría Política en Madrid. Ha realizado más de 40 consultorías en comunicación para organismos internacionales y multilaterales tales como PNUD, ONU Mujeres, OEA, IDEA Internacional, y NIMD. Conferencista regular en foros internacionales, en 2018 fue invitada al Parlamento Europeo para presentar una agenda para el fortalecimiento del liderazgo político de las mujeres. Autora de 11 libros. Su título más reciente ¿Quién teme el Poder de las Mujeres? Bailar hacia atrás con tacones altos fue publicado en España, y también en inglés con el título Dancing Backwards in High Heels. Women, Leadership and Power. virgarcia@sociales.uba.ar

Índice H: 18.

Orcid ID: https://orcid.org/0000-0003-2428-8056

Google Scholar: https://scholar.google.es/citations?user=mgkx788AAAAJ\&hl=es\&oi=sra

\section{Orlando D'Adamo}

Doctor en Psicología. Profesor titular de Opinión Pública en la Carrera de Ciencia Política, Facultad de Ciencias Sociales, Universidad de Buenos Aires. Profesor titular de Psicología Política y Liderazgo en la Carrera de Ciencia Política de la Facultad de Derecho y Ciencias Sociales de la Universidad de Belgrano, Argentina. Director del Centro de Opinión Pública de la Universidad de Belgrano. Profesor en los programas de Comunicación Política de la Universidad Autónoma de Barcelona, la Universidad Complutense de Madrid, la Universidad de Santiago de Compostela junto con el Instituto Universitario Ortega y Gasset en Madrid; el Máster en Asesoramiento de Imagen y Consultoría Política de la Universidad Camilo José Cela de Madrid, y la especialización en Comunicación Política en la Universidad de Salamanca, España. Conferencista habitual en universidades de España y América Latina. Ha publicado 10 libros y numerosos artículos en el área de la comunicación política.

orlando.dadamo@ub.edu.ar

Índice H: 18.

Orcid ID: https://orcid.org/0000-0003-4871-6633

Google Scholar: https://scholar.google.es/citations?user=IPaHMi4AAAAJ\&hl=es

\section{Salomé Berrocal Gonzalo}

Doctora por la Universidad Complutense de Madrid y Catedrática de Periodismo en la Universidad de Valladolid (España). Es coordinadora del GIR NUTECO (Grupo de Investigación Reconocido en Nuevas Tendencias en Comunicación). Ha publicado más de 50 trabajos de investigación, 7 de ellos son monografías completas. Ha participado en 10 proyectos de investigación financiados en convocatorias públicas nacionales e internacionales, en 4 de ellos como Investigadora Principal. Ha realizado numerosas estancias de investigación, cabe destacar las desarrolladas en instituciones de prestigio mundial como la George Washington University, University of California San Diego, Suffolk University, en Estados Unidos, University of Toronto en Canadá o las universidades de Milán o de la Sapienza, en Italia, entre otras. Sus líneas de investigación se centran en la comunicación política, con especial interés en los últimos años en el análisis del fenómeno del infoentretenimiento político, la participación social en la vida política, comunicación y opinión pública, sociología de la comunicación. Su último libro editado por Tirant Lo Blanch es Politainment: la política espectáculo en los medios de comunicación. 
salomeb@hmca.uva.es

Índice H: 16.

Orcid ID: http://orcid.org/0000-0002-0483-0509

Google Scholar: http://scholar.google.es/citations?user=5O0TLMAAAAJ\&hl=es\&oi=ao

\section{Marina Gavensky}

Licenciada en Psicología. Graduada en el Posgrado en Comunicación Política e Institucional de la Universidad Católica Argentina. Docente en las asignaturas Psicología Social, y Psicología Social y Comunitaria, en la Universidad de Belgrano, Argentina. Ha publicado artículos en la Revista Mexicana de Opinión Pública y Más Poder Local.

gavenskym@gmail.com

Orcid ID: https://orcid.org/0000-0003-0698-4875

Google Scholar: $\underline{\text { https://scholar.google.es/citations?user=RoF2HSgAAAAJ\&hl=es\&oi=ao }}$ 